\title{
Jinwu Jiangu Capsule affects synovial cells in rheumatoid arthritis through PI3K/Akt/mTOR signaling pathway
}

\author{
Qiuyi Wang\#, Xueming Yao\#, Hui Xu, Daomin Lu, Ying Huang, Fang Tang, Lina Xiao $₫$ and \\ Wukai Ma四
}

Department of Rheumatology and Immunology, The Second Affiliated Hospital of Guizhou University of Traditional Chinese Medicine, Guiyang, Guizhou Province, 550001, China

\begin{abstract}
Jinwu Jiangu Capsule is a medicinal formula from the Chinese Miao nationality. Leflunomide is recommended in organizational guidelines for the treatment of rheumatoid arthritis (RA). To investigate the effect of Jinwu Jiangu Capsule on PI3K/Akt/mTOR signal pathway in cells taken from RA patients. New Zealand rabbits were administrated with Jinwu Jiangu Capsule suspension to prepare serum containing medicine. Lyophilized powder was prepared from this serum for cell treatment. The expression of LC3-II and PI3K, Akt, mTOR were detected by IF and western blot. Moreover, the levels of Atg1, Atg5, Atg14 were detected by RT-qPCR. The results showed that the expression of LC3-II was increased, and fluorescence spot of LC3-II was obvious in high-dose of Jinwu Jiangu Capsule group. Jinwu Jiangu Capsule decreased the level of PI3k, Akt, and mTOR protein, and increased the levels of Atg1, Atg5 and Atg14. Specially, the highdose of Jinwu Jiangu Capsule had the most obvious inhibitory and up-regulation effects. However, there was no significant difference in the expression of Akt, mTOR and Atg1 in the medium-dose of Jinwu Jiangu Capsule group compared with the leflunomide group. In conclusion, Jinwu Jiangu Capsule regulates autophagy by inhibiting the PI3K/Akt/mTOR pathway in RA.
\end{abstract}

Keyword: Rheumatoid arthritis; Synovial cells; Jinwu Jiangu Capsule; Autophagy

Received: 10 October, 2020; revised: 09 February, 2021; accepted: 26 May, 2021; available on-line: 27 July, 2021

『e-mail: 1140957096@qq.com (LX); fsmymwk@163.com (WM) \#These authors contributed equally to the work.

Acknowledgements of Financial Support: National Natural Science Foundation of China (No: 81760907 and No: 81760829); Guizhou Province High-level Innovative Talent Training Program"Hundred" Level Talent Project (Guizhou Kehe Platform Talent [2016]5650); National Key Research and Development Program: Research on Modernization of Traditional Chinese Medicine (2017YFC1703904); Guizhou Science and Technology Planning Project (Guizhou Kehe Platform Talent [2020]2202).

Abbreviations: ANOVA, analysis of variance; Akt, protein kinase

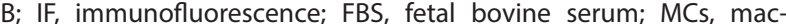
rophage-like cells; mTOR, mammalian target of rapamycin; PI3K, phosphatidylinositol 3-kinase; PFA, paraformaldehyde; PMPs, platelet microparticles RA, rheumatoid arthritis; RA-FLS, RA-fibroblastlike synovial; tid, three times a day; qd, every day; RT-qPCR, quantitative real time polymerase chain reaction; TBST, Tris Buffered saline Tween

\section{INTRODUCTION}

Rheumatoid arthritis (RA) is a common autoimmune disease characterized by persistent synovial hyperplasia, pannus formation and progressive cartilage destruction. RA can cause multiple systemic and multi-organ damage, such as blood, breathing, and cardiovascular damage. RA has a long course, high disability rate and low cure rate. However, the pathogenesis of RA is still unclear.

Autophagy is a complex cellular mechanism that maintains the homeostasis and integrity of cells and tissues by degrading senescent or defective organelles, infection factors, and misfolded proteins (Kroemer, 2015). The regulatory mechanism of autophagy involves the expression of multiple related proteins and signaling pathway transduction. The phosphatidylinositol 3-kinase (PI3K), protein kinase B (Akt) and mammalian target of rapamycin (mTOR) signaling pathway are widespread in synovial tissues as important negative regulatory pathways, and have significant impact on the proliferation of RA-fibroblast-like synovial (RA-FLS), osteoclast activation and synovial angiogenesis (Kim et al., 2012; Wu et al., 2017; Yamaguchi et al., 2019).

Jinwu Jiangu Capsule is a medicinal formula from the Chinese Miao nationality and follows the theory of "trinity" of Miao medicine. In Jinwu Jiangu Capsule, Cibotium barometz is the leading medicine, which has the effect of dispelling wind-dampness, tonifying the liver and kidney, and strengthening the backbone. As a guardian medicine, insect and ant drugs of Zaocys can dispel wind and stop spasm, acting directly on the diseased area. Homalomena occulta, Sabia parviflora Wall. ex Roxb., and Gardneria angustifolia Wall. dispels the wind and strengthens the muscles and bones. Carcuma longa and Panaxnotoginseng (Burk.) F.H.Chen promote blood circulation and relieve pain, while Paeonia lactiflora Pall. and Glycyrrbiza uralensis Fisch. soften the tendons and relieve spasm. The above-mentioned seven drugs are foundation drugs, which can strengthen the function of the leading drug and assist in the treatment of secondary symptoms. Jinwu Jiangu Capsule has the effect of tonifying the kidney and activating blood, dispelling wind, and dampness, and relieving pain. Preliminary study has shown that Jinwu Jiangu Capsule has good clinical efficacy in relieving joint pain and improvement of inflammation (Ma et al., 2016; Yuan \& Ma, 2018). Therefore, this experiment further explored the mechanism of Jinwu Jiangu Capsule in the treatment of RA from the PI3K/ Akt/mTOR signaling pathway that is dependent on autophagy, in order to provide theoretical and experimental evidence for the clinical medication of RA.

\section{MATERIALS AND METHOD}

\section{RA-FLS sample collection}

Synovial tissues were derived from patients with active RA who underwent artificial knee arthroplasty or ar- 
throscopy at the Second Affiliated Hospital of Guizhou University of Traditional Chinese Medicine and Guiyang Orthopedic Hospital. The diagnosis of RA patients met the RA classification criteria (Aletaha et al., 2010), and was confirmed by postoperative pathology. All experimental procedures and protocols were approved by the Medical Ethical Committee of Guizhou University of Traditional Chinese Medicine. This study obtained informed consent from all patients.

\section{Cell culture}

The synovial tissues were rinsed 3 times in PBS to remove blood and fat, cut into pieces not exceeding $1 \mathrm{~mm}^{3}$, and then arranged them evenly, and placed dots on the bottom of the culture flask. The appropriate amount of complete culture medium was added into the culture flask and the tissues were incubated in $37^{\circ} \mathrm{C}$ in an incubator with $5 \% \mathrm{CO}_{2}$ for $3-4 \mathrm{~h}$. According to the growth of cells and the color of the culture medium, the medium was changed every 2-4 d. When the cell confluence reached 80-90\%, 0.25\% trypsin (HyClone, USA) was used to digest and passage, and 3-5 generations of relatively purified cells were used for subsequent experiments.

\section{Preparation of medicated serum}

Twelve clean New Zealand white rabbits (half male and half female, weight $2 \pm 0.2 \mathrm{~kg}$ ) were obtained from Animal Experiment Center of Guiyang Medical College (Guiyang, China). The rabbits were acclimatized for 1 week prior to the experiment and were fed freely with food and water. The feeding environment was kept quiet, hygienically clean, well-lit, and the temperature controlled at $20-25^{\circ} \mathrm{C}$. The rabbits were randomly divided into three groups: the control group, the high-dose of Jinwu Jiangu Capsule group, and the leflunomide group. The drug dose for rabbits was converted to the usual daily dose of $60 \mathrm{~kg}$ adult. The adult dosage is $1.8 \mathrm{~g}$ three times a day (tid) of Jinwu Jiangu Capsule and $20 \mathrm{mg}$ every day (qd) of leflunomide. In the high-dose of Jinwu Jiangu Capsule group, the rabbits were given a gavage with $2700 \mathrm{mg} / \mathrm{kg}$ suspension of Jinwu Jiangu Capsule (the Second Affiliated Hospital of Guizhou University of Traditional Chinese Medicine, China) (Ma et al., 2017). In the leflunomide group, the rabbits were given a gavage with $1 \mathrm{mg} / \mathrm{kg}$ suspension of Leflunomide Tablets (SuZhou Changzheng Pharmaceutical Factory, China). In the control group, the rabbits were given a gavage with equal volume of saline. Each rabbit was given medicine twice a day for 3 consecutive days. Blood was collected from the heart under aseptic conditions $1 \mathrm{~h}$ after the last administration. Then, the serum was separated from blood, sterilized with $0.22 \mu \mathrm{m}$ microporous membrane, and vacuum dried at $-80^{\circ} \mathrm{C}$.

The separated serum was lyophilized step by step, dried under vacuum at $-80^{\circ} \mathrm{C}$, evaporated to dry powder, and stored at $4^{\circ} \mathrm{C}$.

\section{Cell grouping}

Cells in the logarithmic growth phase were digested by $0.25 \%$ trypsin, seeded in 6 -well plates with the density of $5 \times 10^{5}$ cells/well, and cultivated at $37^{\circ} \mathrm{C}$ in an incubator with $5 \% \mathrm{CO}_{2}$ until the confluence reached $80-$ $90 \%$. Then, cells were divided into six groups: control group, rabbit serum group, leflunomide group and high, medium, and low dose of Jinwu Jiangu Capsule group. Cells in the control group were cultured in complete me- dium containing 10\% fetal bovine serum (FBS, Gibco, USA). Cells in the rabbit serum group were cultured in complete medium containing $10 \%$ normal rabbit serum freeze-dried powder from the control group. Cells in the leflunomide group were treated with $10 \mathrm{~g} / \mathrm{mL}$ leflunomide suspension configured with complete medium. Cells in the high, medium, and low dose of Jinwu Jiangu Capsule group were treated with $0.06 \mathrm{mg} / \mathrm{mL}, 0.6$ $\mathrm{mg} / \mathrm{mL}$ and $6.0 \mathrm{mg} / \mathrm{mL}$ Jinwu Jiangu Capsule suspension (Ma et al., 2017). All cells were incubated for $24 \mathrm{~h}$ at $37^{\circ} \mathrm{C}$ in an incubator with $5 \% \mathrm{CO}_{2}$ and collected for follow-up test.

\section{Quantitative real time polymerase chain reaction (RT- qPCR)}

Total RNA from each group of cells was extracted by Trizol (Aidlab, China) according to reagent operation requirements. cDNA was obtained using TIANScript ${ }^{\mathrm{RT}}$ Kit (SYBR Green, China) for reverse transcription and was as a template for RT-qPCR. The reaction conditions were: $95^{\circ} \mathrm{C} 15 \mathrm{~min} ; 95^{\circ} \mathrm{C} 10 \mathrm{~s}, 58^{\circ} \mathrm{C} 30 \mathrm{~s}, 72{ }^{\circ} \mathrm{C}$ $30 \mathrm{~s}$, a total of 40 cycles. The sequence of primers was as follows: GAPDH (F: 5'-ACTCCCAT'TCT'TCCACCTT'TG-3', R: 5'-CCCTGT'TGCTGTAGCCATAT'T-3'), Atg1 (F: 5'-GTTGGTCAAGATCATCCGGC-3', R: 5'-TTC'T'TGGGAGGCATA GACCA-3'), Atg5 (F: 5'-TGAACGAGAAGCAGAGCCAT-3', R: 5'-GATGT TCCAAGGCAGAGCTG-3') and Atg14 (F: 5'-GCAGCGACACCAAGAGAAAA-3', R: 5'-GGTCCCTCCCAGAAGTCTTC-3'). The relative expression of Atg1, Atg5 and Atg14 was calculated by using $2^{-\Delta \Delta C t}$ method.

\section{Western blot}

The total protein was extracted from each group of cells, and denatured in boiling water for $5 \mathrm{~min}$, and separated using corresponding concentration gel. The anti-PI3k (ab154598, ABCAM, UK) was transferred membrane for $3 \mathrm{~h}$. The anti-AKT (ab179463, ABCAM) was transferred membrane for $16 \mathrm{~min}$. The anti-mTOR (ab25880, ABCAM) was transferred membrane for $7 \mathrm{~h}$. After membrane transfer was complete, the protein was blocked for $1 \mathrm{~h}$ at room temperature. The membranes were incubated with primary antibodies at $4^{\circ} \mathrm{C}$ overnight, washed in Tris Buffered saline Tween (TBST) three time, and re-incubated with secondary antibody for $2 \mathrm{~h}$. The bands of membrane were exposed and developed using ECL chemiluminescent liquid, the image was scanned, and the results were analyzed.

\section{Immunofluorescence (IF)}

Synovial cells in the logarithmic growth phase were stained in 4\% paraformaldehyde (PFA, Boster, China) for $30 \mathrm{~min}$, treated with $0.1 \%$ Triton X-100 solution and blocked in 4\% bovine serum albumin (BSA, Boster). After removing the blocking solution, anti-LC3-II was added to the cells (no.3868, Cell signaling Technology, USA) and they were incubated at $4^{\circ} \mathrm{C}$ overnight. After washing with PBS, secondary antibodies were added to the cells, and they were incubated for $1 \mathrm{~h}$ at a room temperature and in the dark. Then, cells were stained with DAPI, washed with the dye nucleus solution, mounted with anti-fluorescence quencher dropwise, and observed and photographed under a fluorescence microscope.

\section{Statistical analysis}

Each group of experiments was repeated at least three times. The data were analyzed by SPSS 23.0 statistical 


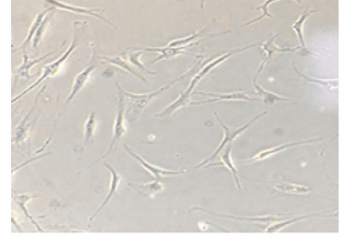

Primary cells $\times 200$

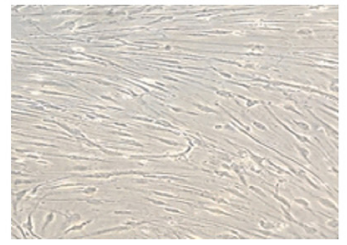

Passage 2 cells $\times 200$

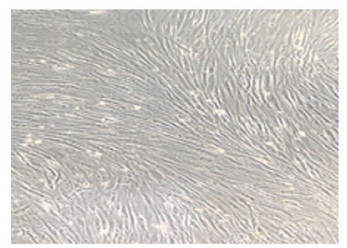

Passage 4 cells $\times 200$

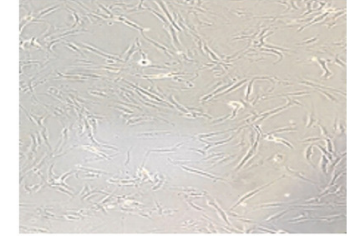

Passage 1 cells $\times 200$

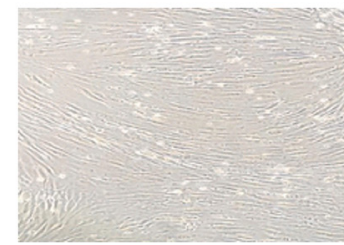

Passage 3 cells $\times 200$

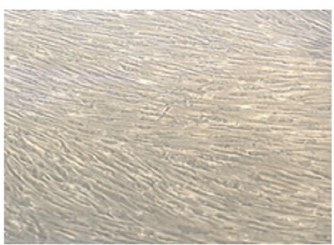

Passage 5 cells $\times 200$
Figure 1. Morphological observation of RA synovial cells. The synovial tissues were derived from RA patients and analyzed by IF.

software (SPSS Inc., USA), and the measures were expressed as mean \pm standard deviation (S.D.) with t-test and analysis of variance (ANOVA). $P<0.05$ indicated that the difference was statistically significant.

\section{RESULTS}

\section{Morphological observation of synovial cells}

Synovial tissues are divided into a lining layer and a sub-lining layer. The lining layer mainly consists of FLS and macrophage-like cells (MCs). The round-shaped MCs do not have regenerative capacity. Thus, the transmission of MCs is gradually disappearing with the digestion of cells, and then gradually purified into FLS of uniform size. After about 2-3 days, the primary cells in Fig. 1 were cultured by the tissue explant method to crawl out from the edge of the tissue, adhere to the wall and extend outgrowths. The microscopic results showed that these cells exhibit a variety of morphologies, predominantly long fusiform, less polygonal, dendritic, and round. The cellular nucleus is oval or elliptical with welldefined boundaries and located in the center of the cell, with obvious nucleoli. Subsequently, the cells showed intensive growth, their proliferation rate was accelerated and were almost purified into a single FLS after three generations of reproduction (Fig. 1). At this time, cells are shuttle-shaped, strongly refractive, spiral or radially arranged under the microscope. Therefore, culturing cells by tissue explant method can avoid the damage to synovial cells by chemical reagents compared with the enzymatic digestion method and yields cells with typical morphology and fewer heterogeneous cell types.
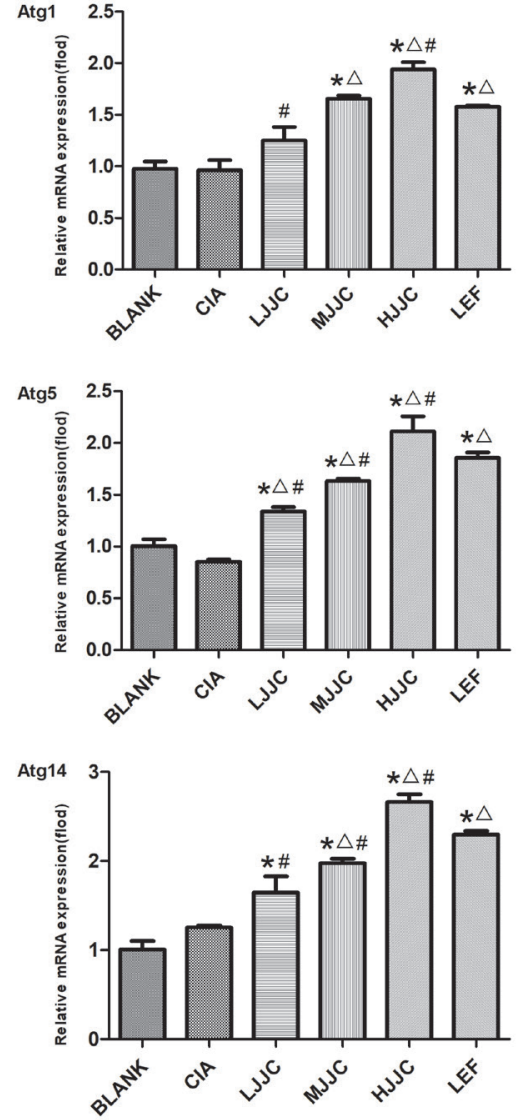

Figure 2. Jinwu Jiangu Capsule up-regulated the expression of Atg1, Atg5 and Atg14 in RA-FLS cells.

The mRNA expression of Atg1, Atg5 and Atg14 in RA-FLS cells were detected by RT-qPCR. ${ }^{*} P<0.01$ represented that Jinwu Jiangu Capsule group compared with control group; $\triangle P<0.01$ represented that Jinwu Jiangu Capsule group compared with rabbit serum group; ${ }^{*} P<0.01$ represented that Jinwu Jiangu Capsule group compared with leflunomide group.

\section{Jinwu Jiangu Capsule up-regulated the expression of Atg1, Atg5 and Atg14 in RA-FLS cells}

Results in Fig. 2 showed that the mRNA expression of Atg1, Atg5 and Atg14 in Jinwu Jiangu Capsule group was significantly increased compared with control group or rabbit serum group $(P<0.01)$. There was no significant difference in the mRNA expression of Atg1 in the medium-dose of Jinwu Jiangu Capsule group compared with the leflunomide group $(P=0.503)$. However, the high-dose of Jiangu Capsule group had stronger up-regulation of Atg1, Atg5, and Atg14 expression $(P<0.01)$.

\section{Jinwu Jiangu Capsule reduced the protein activity of PI3k, Akt, and mTOR in RA-FLS cells}

As shown in Fig. 3, the expression levels of PI3k, Akt and mTOR protein in Jinwu Jiangu Capsule group were decreased than that in control group or rabbit serum group $(P<0.01)$. Moreover, PI3k, Akt and mTOR protein expression was down-regulated in the high-dose of Jiangu Capsule group rather than that in the leflunomide group $(P<0.01)$. But the medium-dose of Jiangu Capsule group and the leflunomide group had similar down-regulation effects on Akt and mTOR expression. 

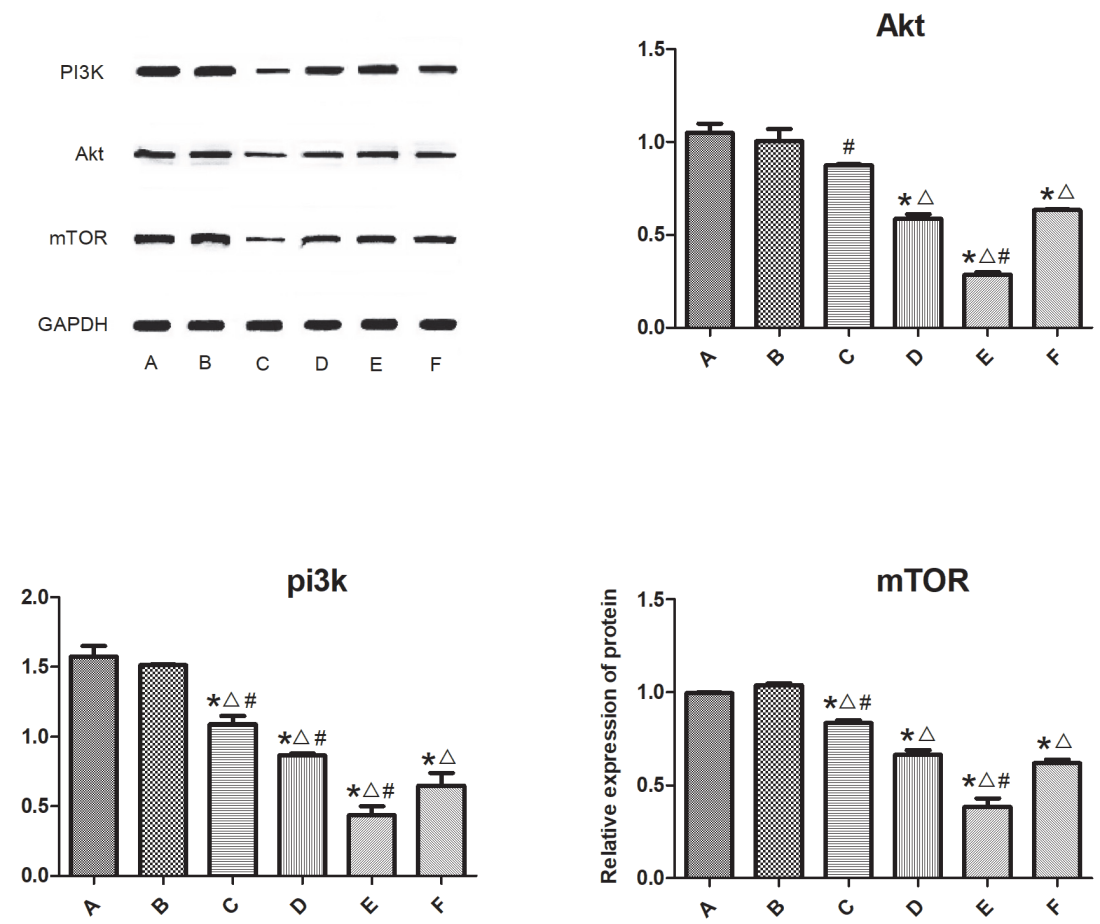

Figure 3. Jinwu Jiangu Capsule reduced the protein activity of PI3k, Akt, and mTOR in RA-FLS cells.

The expression of PI3k, Akt, and mTOR protein in RA-FLS cells were detected by western blot. ${ }^{*} P<0.01$ represented that Jinwu Jiangu Capsule group compared with control group; $\Delta P<0.01$ represented that Jinwu Jiangu Capsule group compared with rabbit serum group; $\# P<0.01$ represented that Jinwu Jiangu Capsule group compared with leflunomide group.

\section{Jinwu Jiangu Capsule increased LC3-II expression in RA-FLS cells}

LC3 is an important marker for autophagosome formation and is diffusely distributed in the cytoplasm at rest. Once autophagy is activated, LC3-I in the cytoplasm will be translocated to the autophagosome membrane to form LC3-II, so the cells that undergo autophagy can be identified by fluorescently labeled LC3-II. In Fig. 4, the number of LC3-II fluorescence spots increased to varying degrees after RA-FLS cells was treated with Jinwu Jiangu Capsule, and the spot phenomenon was obvious in the high-dose of Jinwu Jiangu Capsule group.

\section{DISCUSSION}

mTOR is an important confluence point for cellular autophagy regulated by multiple signaling pathways. It is activated by changes in upstream regulatory factors, such as loss of negative regulator PTEN, abnormalities in TSC complex or activation of PI3K/Akt pathway. PI3K exists in various cytoplasms and is classified into three classes (Type I, II and III) based on the specificity of its substrate. Type $\mathrm{I}$ is a heterodimer structure composed of catalytic subunits $(\mathrm{p} 110 \alpha, \beta, \gamma, \delta)$ and regulatory subunits $(\mathrm{p} 85 \alpha, \beta, \gamma)$ (Ito et al., 2018). Type I of PI3K translocates to the plasma membrane when the extracellular ligand binds to the corresponding receptor, and further catalyzes PIP2 to produce PIP3 in order to recruit signal proteins that carry PH domains. Among these proteins, Akt negatively regulates autophagy by inhibiting the TSC1/TSC2 protein complex to put Rheb in a GTPbound state to enhance mTORC1 activity, which in turn promotes the expression of apoptosis-related proteins and inhibits the occurrence of ULK1 complex (Koren et al., 2010; Antonioli et al., 2017).

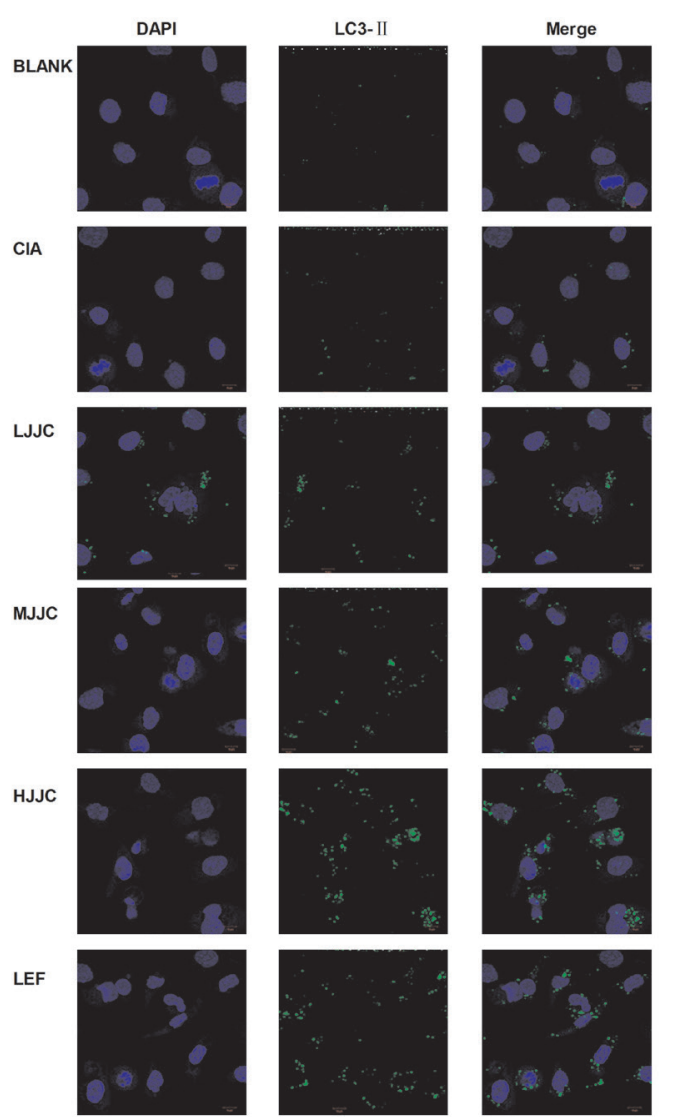

Figure 4. Jinwu Jiangu Capsule increased LC3-II expression in RA-FLS cells.

The fluorescent label of LC3-II was detected by immunofluorescence (IF). 
Atg1 is a key mediator of mTORC1 signaling to autophagy, which regulates the early stage of autophagosome formation when the external environment is altered or mTORC1 is inactivated. Upon autophagy induction, Atg14 constitutes the type I of PI3K complex that aggregates to the junction of endoplasmic reticulum and mitochondria, recruiting relevant effector molecules and then nucleate (Zhao et al., 2014). The polysome Atg12Atg5-Atg16L contributes to the elongation of the isolated membrane and also promotes autophagosome maturation, allowing LC3-I to target its lipid-binding membrane site (Malhotra et al., 2015). In addition, Atg5 has a role in promoting apoptosis (Shi et al., 2013; Messer \& Sciences 2017; Song et al., 2017). FLS is the major effector of RA joint destruction and inflammation. It can migrate and invade cartilage and bone, accelerate synovial hyperplasia and pannus formation. Autophagy promotes cell survival under stress conditions, and apoptosis is the basic mechanism of programmed cell death. Aberrant activation of PI3K-AKT signaling in RA-FLS cells leads to high expression of downstream anti-apoptotic genes (Perlman et al., 2000). In addition, PI3K accelerates the cell cycle by Akt/mTOR transmits mitogenic signals to the ribosome S6 protein kinase (p70s6k), causing continuous proliferation of RA-FLS cells (Ito et al., 2018). The above phenomenon stimulate the increase of oxygen consumption in the joint cavity, and the hypoxic microenvironment promotes the formation of synovial neovascularization to increase oxygen supply (Kim et al., 2015; Fearon et al., 2016). Studies have confirmed that PI3K and Akt are closely related to the release process of platelet microparticles (PMPs) (Franca et al., 2014; Su et al., 2016). The levels of PMPs are significantly elevated in joint fluid and peripheral blood of RA patients and regulate microvascular neovascularization by recruiting receptors for platelet adhesion molecules to induce endothelial cell proliferation and migration (Knijffdutmer et al., 2002).

Guizhou is located on the Yunnan-Guizhou Plateau with humid and cold climate. Guizhou is prone to a high incidence of RA due to the fact that its inhabitants were fond of acidic and perishable foods, which tend to cause pain and stiffness in the muscles and veins. Jinwu Jiangu Capsule is a Chinese medicine based on the experience gained from years of treatment and is prepared formulated in accordance with the "trinity" of Miao Medicine. Jinwu Jiangu Capsule is effective in replenishing renal, vivifying blood flow, dispelling wind and eliminating dampness, and activating meridians to stop pain. Jinwu Jiangu Capsule has a synergistic effect of a variety of herbs and a multi-faceted combination of drugs, with a transfer of medicinal effects and a precise combination. The previous study confirmed that Jinwu Jiangu Capsule has good therapeutic effect on RA.

In this study, we used the tissue explant method to culture knee FLS cells in patients with RA and selected three to five generations for experimental testing. The results of the study showed that Jinwu Jiangu Capsule acts on RA-FLS cells to inhibit the expression levels of PI3K, Akt and mTOR proteins. It was further found that LC3-II showed punctate aggregation under the microscope after the intervention of Jinwu Jiangu Capsule, and its fluorescence signal showed an enhanced trend. At the same time, the mRNA level of autophagy-related genes Atg1, Atg5, and Atg14 was also significantly increased in RA-FLS cells. Moreover, the up-regulation of Atg1, Atg5, and Atg14 expression was enhanced with increasing doses of Jinwu Jiangu Capsule and was more pronounced in the high-dose group. Meanwhile, the upregulation changes in the medium-dose of Jinwu Jiangu Capsule group were similar to those in the leflunomide group.

In conclusion, Jinwu Jiangu Capsule could regulate cell autophagy to control the development of RA through inhibiting $\mathrm{PI} 3 \mathrm{~K} / \mathrm{AKT} / \mathrm{mTOR}$ signaling pathway and ultimately achieve the goal of clinically reducing synovial inflammation and relieving joint pain and swelling in patients with RA. The present study will provide new drug and clinical treatment options for the treatment of RA.

\section{Acknowledgements}

Not applicable.

\section{Competing interests}

The authors state that there are no conflicts of interest to disclose.

\section{Ethics approval}

All experimental procedures and protocols were approved by the Medical Ethical Committee of Guizhou University of Traditional Chinese Medicine.

\section{Statement of Informed Consent}

Written informed consent was obtained from a legally authorized representative(s) for anonymized patient information to be published in this article.

\section{Availability of data and materials}

All data generated or analyzed during this study are included in this published article.

\section{Authors' contributions}

Qiuyi Wang and XuemingYao designed the study, supervised the data collection, Hui $\mathrm{Xu}, \mathrm{Daomin} \mathrm{Lu}$ and Ying Huang analyzed the data, interpreted the data, Fang Tang, Lina Xiao and Wukai Ma prepare the manuscript for publication and reviewed the draft of the manuscript. All authors have read and approved the manuscript.

\section{REFERENCES}

Aletaha D, T Neogi, AJ Silman, J Funovits, DT Felson, CO Bingham III, NS Birnbaum, GR Burmester, VP Bykerk,MD Cohen (2010) 2010 rheumatoid arthritis classification criteria: an American College of Rheumatology/European League Against Rheumatism collaborative initiative. Arthritis Rheumatism 62: 2569-2581. https://doi. org/10.1136/ard.2010.138461

Antonioli M, Di Rienzo M, Piacentini M, Fimia GM (2017) Emerging mechanisms in initiating and terminating autophagy. Trends Biochem Sci 42: 28-41. https://doi.org/10.1016/j.tibs.2016.09.008

Fearon U, Canavan M, Biniecka M, Veale DJ (2016) Hypoxia, mitochondrial dysfunction and synovial invasiveness in rheumatoid arthritis. Nat Rev Rheumatol 12: 385-397. https://doi.org/10.1038/nrrheum.2016.69

França CN, Izar MC, Amaral JB, Tegani DM, Fonseca FA (2015) Microparticles as potential biomarkers of cardiovascular disease. Arq Bras Cardiol 104: 169-174. https://doi.org/10.5935/abc.20140210

Ito Y, Hart JR, Vogt PK (2018) Isoform-specific activities of the regulatory subunits of phosphatidylinositol 3-kinases - potentially novel therapeutic targets. Expert Opin Ther Targets 22: 869-877. https:// doi.org/10.1080/14728222.2018.1522302

Kim H-R, Kim K-W, Kim B-M, Cho M-L, Lee S-H (2015) The effect of vascular endothelial growth factor on osteoclastogenesis in rheumatoid arthritis. PLoS ONE 10: e0124909. https://doi.org/10.1371/ journal.pone.0124909

Kim T-H, Choi SJ, Lee YH, Song GG, Ji JD (2012) Combined therapeutic application of $\mathrm{mTOR}$ inhibitor and vitamin D3 for inflam- 
matory bone destruction of rheumatoid arthritis. Med Hypotheses 79: 757-760. https://doi.org/10.1016/j.mehy.2012.08.022

Knijff-Dutmer EA, Koerts J, Nieuwland R, Kalsbeek-Batenburg EM, van de Laar MA (2002) Elevated levels of platelet microparticles are associated with disease activity in rheumatoid arthritis. Arthritis Rheum 46: 1498-1503. https://doi.org/10.1002/art.10312

Koren I, Reem E, Kimchi A (2010) Autophagy gets a brake: DAP1, a novel mTOR substrate, is activated to suppress the autophagic process. Autophagy 6: 1179-1180. https://doi.org/10.4161/ auto.6.8.13338

Kroemer G (2015) Autophagy: a druggable process that is deregulated in aging and human disease. The J Clin Invest 125: 1-4. https://doi. org/10.1172/JCI78652

Ma W, Fan Y, Li Y, Yao X, Qiao Y, Huang Y, Zhou J (2017) Study on the anti-inflammatory effect of total extracts of Jinwu Jiangu Formula on synovial cells in rheumatoid arthritis. J Guiyang College Tradit Chin Med 39: 58-61 (in Chinese)

Ma W, Lu D, Yao X, Huang X, Tang F, Liang J, An Y, Zhou J (2016) Effect of Jinwu Jiangu Formula on the expression of $\mathrm{NF}-x \mathrm{~B}$ and IL-17 in a rat model of collagen-induced arthritis. Chin J Integ Med 36: 0624-0628 (in Chinese)

Malhotra R, Warne JP, Salas E, Xu AW, Debnath J (2015) Loss of Atg12, but not Atg5, in pro-opiomelanocortin neurons exacerbates diet-induced obesity. Autophagy 11: 145-154. https://doi.org/10.108 $0 / 15548627.2014 .998917$

Messer JS (2017) The cellular autophagy/apoptosis checkpoint during inflammation. Cell Mol Life Sci 74: 1281-1296. https://doi. org/10.1007/s00018-016-2403-y

Perlman H, Georganas C, Pagliari LJ, Koch AE, Haines K 3rd, Pope RM (2000) Bcl-2 expression in synovial fibroblasts is essential for maintaining mitochondrial homeostasis and cell viability. I Immunol 164: 5227-5235. https://doi.org/10.4049/jimmunol.164.10.5227

Shi M, Zhang T, Sun L, Luo Y, Liu DH, Xie ST, Song XY, Wang GF, Chen XL, Zhou BC, Zhang YZ (2013) Calpain, Atg5 and Bak play important roles in the crosstalk between apoptosis and autophagy induced by influx of extracellular calcium. Apoptosis 18: 435-451. https://doi.org/10.1007/s10495-012-0786-2

Song S, Tan J, Miao Y, Li M, Zhang Q (2017) Crosstalk of autophagy and apoptosis: Involvement of the dual role of autophagy under ER stress. J Cell Physiol 232: 2977-2984. https://doi.org/10.1002/ jcp. 25785

Su XL, Su W, Wang Y, Wang YH, Ming X, Kong Y (2016) The pyrrolidinoindoline alkaloid Psm2 inhibits platelet aggregation and thrombus formation by affecting PI3K/Akt signaling. Acta Pharmacol Sin 37: 1208-1217. https://doi.org/10.1038/aps.2016.52

Wu X, Long L, Liu J, Zhang J, Wu T, Chen X, Zhou B, Lv TZ (2017) Gambogic acid suppresses inflammation in rheumatoid arthritis rats via PI3K/Akt/mTOR signaling pathway. Mol Med Rep 16: 7112 7118. https://doi.org/10.3892/mmr.2017.7459

Yamaguchi K, Sudo H, Imai K (2019) Vascular endothelial growth factor signaling in VE-cadherin expression and tube-like formation by rheumatoid arthritic synovial fibroblast-like cells. Biochem Biophys Res Commun 508: 405-409. https://doi.org/10.1016/j.bbrc.2018.11.116

Yuan X, Ma W (2018) Effectiveness of Jinwu Jiangu formula in the treatment of rheumatoid arthritis. Asia-Pacific Traditional Medicine. 14: 188-190 (in Chinese)

Zhao Z, Tao L, Shen C, Liu B, Yang Z, Tao H (2014) Silencing of Barkor/ATG14 sensitizes osteosarcoma cells to cisplatin-induced apoptosis. Int J Mol Med 33: 271-276. https://doi.org/10.3892/ ijmm.2013.1578 